\title{
Infant Brain Development And The Impact Of Breast Feeding: A Review Of Literature
}

Molly H. Minkkinen, (Email: mminkkin@d.umn.edu), University of Minnesota Duluth

\begin{abstract}
Research in the area of infant development has endless facets of investigation. No one facet of research is more important than another, and all of the findings work in a synchronous fashion to facilitate our understanding of child development. Research on child development has proliferated across the centuries. Infant characteristics like attention, digestion, and behavior are among the areas studied. The research findings in these and many other areas have deepened our understanding of how infants respond to their environments, and to the people who care for them. The findings from past research have also led to changes in strategies for interacting with infants in the medical, educational, and parenting realms.
\end{abstract}

\section{INTRODUCTION}

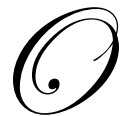

ne area of research interest in the past decade has focused on the issue of infant brain development. In most cases, the goal of the research has been to attempt to answer long-standing questions that past scientists and researchers were prevented from studying, because of a lack of technological advancement and expertise. The questions focused on the biological function of cognition, how chemical reactivity influences brain function, the biological origin of temperament, and the biological impact of breast feeding on early brain development. Advancements in science and technology, along with the work of past researchers, have led to a time when many hard-to-answer questions about infant brain development are ripe for investigation. Now is the time when researchers can investigate the role breast feeding has on the biological function of the brain and the subsequent temperament of the infant.

The purpose of this paper is to create a clear picture of the work that has been done by previous researchers in the areas of infant development and neurobiology by reviewing literature in these areas. The review of literature is intended to provide an overview of past and current research findings, which will lead to an understanding of current theories of infant development in the area of neurobiology as well as uncover the relationship between brain development and breast feeding and look at how temperament plays a part in the overall development of the infant brain. This understanding is intended to set forth a direction for further research.

In order to create a comprehensive picture of these issues, this paper will review literature in four separate parts and conclude with syntheses of the findings. Part one will be a review of literature related to early brain development. Part two will review the chemical substrates of the brain. Part three will review theories of temperament. The final section will review the role breast feeding plays in a child's development.

\section{EARLY BRAIN DEVELOPMENT}

Allen Schore (2001) states that the greatest amount of neurobiological research is on the adult rather than the developing brain, and most of it is not on normal but on abnormal brain function. Even so, contemporary neuroscience is now beginning to become interested in the early developmental failures of the brain. As a result, neurobiology is currently exploring early beginnings and brain pathology (Schore, 2001). 
Pediatric neurologist Harry Chugani who studies early brain development, notes, "There is no doubt that experience molds the young brain. The early years determine how the brain turns out” (Schore, 2001, p. 1). He goes on to make clear that the child's potential is determined in the early years-from the first moment of life to countless hours spent in day care. He says, "These are the years when we create the promise of a child's future. This is when we set the mold" (Schore, 2001, p. 1). Many scientists have said that kindergarten is not the starting point of the child's brain development. They say that by kindergarten the process is half over (Schore, 2001).

Although the work of Schore Chugani and others make clear the obvious movement today in the sciences to research early brain development, there is a well-paved road to the current practice of child care and neurobiology that is rich in history.

In an article titled "Neonatal Neurology, Past Present and Future: A Window on the Brain," the author, Lily Dubowitz and her colleagues describe the historical journey of the study of infant brain development. Dubowitz, Cowan, Rutherford, Mercuri, and Pennock (1995) state that the first examination of the brain had taken place in the 1940s and 50s. The study of the brain at the time was aimed at evaluating the neurological state of the full term infant. The first examination was developed by Andre' Thomas and Saint-Anne Dargassies (1952). The examination mainly involved the evaluation of passive and active tones and defined in detail various primitive reflexes. The findings of the examinations mapped out the maturation of the neurological features in premature infants of various gestational ages and were able to show that the development characterized related to the maturity and not the size of the infant.

Dubowitz et al. (1995) write further about the history of infant brain research by citing the work of Prechtl (1977) of Holland. Prechtl's research followed that of Thomas and Dargassies. In the 1970s Prechtl developed an examination which concentrated on the neurological responses of the full-term infant. The aim of the research was to take into account the behavioral state of the newborn. The findings were able to demonstrate that many of the neurological signs in a newborn were very much dependent on the state of alertness at the time the newborn was evaluated.

Dubowitz et al. (1995) continue their review of historical research in the area of infant brain development with the work of T. Berry Brazelton in the early 1970s. Brazelton developed a behavioral examination, which was based largely on an earlier examination developed by K.F. Graham [check the initials - they are different in the reference section] in the 1950s. The items included tests that measured motor activity and strength, responsiveness to auditory stimuli as well as measured irritability and tension in infants. It also aimed to evaluate the interaction between the newborn infant and its caretakers (Dubowitz et al., 1995).

Dubowitz et al. (1995) indicated that their research findings suggest that there were problems with the neurological examinations of the past, because the examinations were generally poor in predicting outcomes. The researchers in Dubowitz's team also note that "while it was possible to relatively easily identify infants with normal outcomes as those who were persistently normal in the neonatal period, there were large number of infants who showed abnormalities in the newborn period yet had completely normal outcomes later on” (pg 23).

The work of Thomas and Dargassies (1952), Prechtl (1977), Brazelton (1972), and others were successful in creating an understanding of the human brain function, which has, in turn, provided insight into early brain development. The synthesis of their work has identified behavioral benchmarks in brain development that show stage and phase related consistencies among developing infants. The measures, although empirical, left questions that were technologically unanswerable.

The advancements that have occurred recently in science and technology have created measures that have the capability of looking at the biological structure of the brain such as magnetic imaging and magnification. These technological advances have been instrumental in identifying the architectural structures of the developing brain. The information gathered from these measures has added depth to our understanding of how young brains organize, function, and development neurologically. Dubowitz et al. (1995) describe that data that was instrumental in this theory development in their article entitled "Neonatal Neurological, Past, Present and Future A Window on the 
Brain”. The article described the data collected by Dubowitz which focused on live infants. The use of technology such as the MRI and the cranial ultrasonography was instrumental in their findings. The Dubowitz et al. study of live infants examined the incidence, timing, and evolution of the lesions in the neonatal period. The researchers were able to follow the evolution of the lesions into infancy. The findings from this work are significant in the development of an understanding of development of brain lesions in live infants. Because these technologies are available and, in the case of the ultrasonography, portable, this understanding is typically referred to as the maturational theory of brain development. Discussions of this theory are significant to the field of early childhood education, child development, and other related fields because they suggest that early neurological brain function is essentially the creation of cognitive constructs that become the function of sight, language, emotional response, and creativity.

Within the confines of the maturational theory the architectural structure of the developing brain has recently been identified. The theory has been developed with the use of new technologies such as brain imaging machines. Three of the most frequently used machines are the functional magnetic resonance imaging (fMRI), positron emission tomography (PET), and magnetic resonance imaging (MRI) machines. Tests using the machines have shown that the early brain architecture is made up of 100 billion neurons. Each neuron has a nucleus, an axon and many branch-like dendrites. Each neuron has the potential to make a synaptic connection with approximately 60,000 to 100,000 other neurons (Pally, 1997). Researchers Gunnar and Barr (1998) describe the development of the architectural process as the biological growth and differentiation of the developing brain. They suggest that the development and migration of the neurons in the developing brain consist of a series of biological events that proceed in a relatively well-ordered but overlapping sequence. This sequence consists in the birth and migration of cells, the growth of the axon, and the formation of the dendrites that allow connections among neurons, the formation of the synapse that allows the information flow across neurons and then the mylenation that speeds up the transmission of neural impulses. The repeated process of this biological sequence is the precursor to the circuit development of the brain that will be discussed later.

Gunnar and Barr state (1998) that axon development is largely complete by birth in full-term infants and dendritic growth, synaptic function, and mylenation are largely postnatal events. Most of the brain growth in these areas has been found to take place during the first two years of the infant's life.

In keeping with the maturational theory, Diamond (1988) suggests that the brain has a use it or lose it structure. She states that we are born with an overabundance of neurons and dendrites, and that a pruning process begins during the prenatal period. This pruning is the result of the experience-dependent circuit development of the neural pathways. The neurons that are used remain; while those that are not die off (Pally, 1997). The neural circuit that Diamond describes is the foundation to the constructs that substantiate learning throughout the infant's life. Gunnar et al. (1998) [is this the 1996 work by Gunner, Brodersen, Nachmias, Buss, and Rigatuso? With 4 works starting with this author, you'll need to double-check them.] indicate that findings from animal research show that early experiences program the brain's neurological circuits in ways that affect later cognitive competence, emotional responding, and activities of physiological systems that orchestrate reactions to stress and challenge.

The architectures that Gunnar and Barr (1998), Diamond (1988), and others describe are the microscopic underpinning of larger structures in the brain. These structures are thought to have a great evolutionary history. According to the MacLean model or the Triune model, developed by P.D. MacLean in the early 1950s there are three distinct yet interrelated parts of the brain that have developed systematically from our ancestors. He suggests that there is a reptilian brain, a lower mammalian brain, and a primate brain (Pally, 1997). This model of brain evolution developed in the 1950s and became popular in the 1970s and 1980s. It is currently viewed by most researchers as an outdated model due to the simplistic categorization of brain function. An understanding of the actual dynamic function of the brain has changed as a result of the research from the recent decade. However, the categories MacLean identified are still thought to be accurate. Because of this, the model is still used as a basic structural view of the brain. The part of the brain that McLean identified as the reptilian brain is currently known as the brain stem. It is responsible for the vital functions of physiological survival such as sleep/wake cycles, heart rate, respiration, and body temperature. MacLean's lower mammalian brain is currently called the limbic or subcortical region of the brain and is thought to have primary control of the emotional function of the brain. This region of the brain is responsible for such things as parental care; including nursing, emotion, memory, play and the infant distress cry (Pally, 1997). What 
MacLean called the primate brain is currently referred to as the cortex. This area of the brain is thought to control the higher order thinking function. This region of the brain is significant to infant brain development in that it modulates the emotion, behavior, and body physiology processed by the lower brain (Pally, 1997). According to MacLean, through natural selection "newer" brain structures, which could perform more adaptive functions, were added on to and integrated with more primitive structures (Pally, 1997).

As mentioned, researchers suggest that the model presents an over simplified view of the brain. The brain is in fact a very dynamic structure that is whole in its function. Despite the current view, I use MacLean's Triune or modified Triune model in this work for two reasons. First, the model provides a simple structural view of the human brain. Secondly, the structures that MacLean identified are not inaccurate. They do exist in the manner in which he describes them. However they are found to be more complex in their function than MacLean originally thought. I believe that for the purpose of structural identification this model is useful.

Using the research findings of MacLean and others, Pally (1997) has conducted extensive research in the area of early brain development that has led to the development of a theory that suggests that the brain's design strikes a balance between a circuit's permanence and a circuit's plasticity. Circuit permanence is the result of repeated exposure to a given stimuli. Many researchers refer to this phenomenon as "hardwiring." As a child has repeated exposure to sight, sound, touch and other stimuli, the neurons responsible for securing an understanding of the situation create a permanent pathway related to that given information. An example of this, for older children, would be riding a bicycle or playing a Mozart concerto (Pally, 1997). As the behavior is repeated, the neural circuits hardwire. The hardwired motor pattern is stored in the basal ganglia in the brain stem and is activated as automatic motor routines (Pally, 1997). Infants have the same developmental process with experiences like vision and parental response. As an infant has repeated visual information, the neural pathways in the occipital region of the brain (primarily responsible for vision) hardwire the pathways that in turn provide visual input. This hardwired neural pathway responds automatically, creating visual capabilities for the infant. Repeated parental response will also result in a hardwiring process in the limbic region of the brain (primarily responsible for emotional reactivity). An infant will respond to parental behavior in a predictable manner based on repeated past responses by the parent. In other words the infant hardwires his/her understanding of the care expected through repeated behavior on the part of the parent. If an infant is picked up, soothed and responded to appropriately, the brain will make neural connections that become "hardwired" and, in turn, the brain will expect the same response in the future. The phenomenon of neural plasticity is quite the opposite of "hardwiring." Neural plasticity describes the flexibility of the neural circuitry to adapt and adjust to stimuli. According to Diamond (Diamond \& Hopson, 1988), neural plasticity is essentially neural flexibility. Diamond (1988) states that neural plasticity has the same functional resilience and pliancy of living silly putty. Diamond goes on to say that "more than any other organ, the brain can be shaped by stimulation and use, by disease, and trauma, by dull routine and disuse into a center of thought, sensation, and regulation most appropriate for a given individual's life" (p. 58). Healthy brain development has the presences of both neural hardwiring and neural plasticity. Pally states (1997) that more plasticity exists in cortical circuits where new dendrites can grow and synaptic connections can continue to be made throughout life. An example would be that cortical functions such as vocabulary and math have a lot of plasticity. People can continue to learn a number of words for the same concept and a number of solutions to a problem. On the other hand, the subcortical limbic "emotional" circuits that develop in infancy have less plasticity and therefore may have a long-lasting effect on subsequent psychological development. It seems that these are the circuits that, through repetitive performance, create permanence that allows children to form and maintain attachments to their parents over the long period of their development and to seek familiar, reliable sources of safety and comfort. As mentioned earlier, through maturation, the cortex develops the capacity to modulate emotional responses of the subcortex, a process that is easily observable as children and adolescents grow (Pally, 1997).

Pally (1997) also points out that the human brain is born prematurely, and that because of this, much of the brain's development occurs postnatally and for many years forward. This development includes both neural hardwiring and plasticity of the neural circuits. The significance of the neural process of hardwiring and plasticity are compounded when considering neural pruning. Diamond (1988) describes the phenomenon of neural pruning in Pally's work related to early brain development. Diamond describes the architecture of the developing brain as having a use it or lose it process. Pally quotes Diamond (1988) as saying that brain development is a matter of "use it 
or lose it." Diamond continues on to say that we are born with an overabundance of neurons and dendrites and during the neonatal period a pruning process begins. As a result of experience-dependent circuit development, the neural paths that are used remain, while those that are not used die off (Pally, 1997) adds to this theory by suggesting that many aspects of brain development are activity dependent (use dependent), and thus incorporate experience as part of the basic program for growing a brain. Diamond says that experiences early in life do appear to influence how the child reacts to later experiences and how the brain processes and profits (or fails to profit) from experiences throughout life.

Johnston (1995) brings yet another perspective. He also supports the maturational theory, but suggests that brain research should focus on the notion that brain development has a progressive nature of maturation from posterior to anterior regions. He cites research findings that indicate that the primary visual cortex is functional shortly after birth, and the frontal lobes and prefrontal cortex are the last parts to become functional as supportive evidence for his theory. Johnson refers to his theory as the cognitive developmental theory and suggests that in the first few weeks after birth many brain pathways are partially activated by task situations in infants, but most of the cortical pathways are under-specialized. He further suggests that the actions of young infants are often based on the output from the first available pathway which may often result in an impoverished or incorrect response when the task situation is more complex or demanding (Johnson, 2000). The theory proposed by Johnson has implications for views on cognitive and behavioral development in infancy. He believes that development is not stage-like in nature and suggests that brain development is dependant on multiple competing representations that are engaged by particular stimuli or task demands, and which compete to influence behavioral output. Johnson's cognitive developmental theory is new and based on the data generated by the author. Johnson (2000) suggests that during the next decade great advances will be made as he comes closer to incorporating evidence from neuroscience into our understanding of mental development in infants and children.

Neurobiologist Michael Posner (2001) looks at infant brain development from a different perspective. He combines the maturational and cognitive developmental theories as he views brain development with a primary interest in relating the human brain to the cognitive and emotional development of infancy and childhood. Posner has focused his research on the behavioral output of the brain and on the attentional networks in the infant brain. In doing so, Posner feels that he is building upon new knowledge about the brain that has developed from the work of maturational researchers as well as recent research in the areas of cognitive development and genetic influences. Posner traced the orientating skills of infancy during the first year of life and found that at four months, infants can learn where to look. The maturational theory suggests that the infants have hardwired their ability to perform visual tracking through repeated visual input. The cognitive theorist would suggest that the infant brain was physically developed enough at four months to perform the task with or without previous experience. Posner (2001) also found that the four-month-old infants did not have the ability to handle ambiguous context and using explicit knowledge to control their behavior development. Gunnar (1996), Diamond (1988), and Pally (1997) suggest that through repeated experience and appropriate social interactions, the behavioral development would emerge. Johnson (2000) suggests that in time the behavioral development would emerge in isolation from interaction and social experience.

In 2001, Allen Schore introduced another view of infant brain development in an article he wrote for the Infant Mental Health Journal_entitled "Contributions From The Decade Of The Brain To Infant Mental Health: An Overview." In his article, Schore outlined a comprehensive theory of infant brain development which he called the psychoneurobiological theory. This theory was developed by integrating areas of study like neurobiology, infant psychiatry, child development, and early childhood education. He states that the psychoneurobiological theory incorporates the findings of many sciences to create a theory about infant brain development and the development of attachment. In discussing his theory, Schore makes clear that findings from this work have contributed to our understanding of child development in general. He points out that his work in the areas of brain development and attachment theory, using a biological perspective, are significant because infant development has long been addressed primarily through the lenses of the psychological sciences. While respecting and retaining the significance of the theories from past work in the areas of infant psychiatry, behavioral pediatrics, and child psychology, Shore, like Pally (1997), Diamond (1988), and Dubowltz (1995), incorporates the work of neuroscience into his theory of infant mental health development. According to Schore (2001), the emergence of the psychoneurobiological model of infant brain development indicates that the work of contemporary neuroscience will now focus on the early developmental growth 
and failures of the brain. He supports this comment by saying that "neurobiology is currently exploring early beginnings and brain pathology” (p. 2).

In creating a case for his theory, Schore identifies two practitioners who have contributed to the field of child development, early childhood studies, and now psychoneurobiology: Daniel Siegel, a pediatric psychologist and pediatrician and John Bowlby, a well-known researcher in the area of attachment theory and infant/caregiver behavior.

Daniel Siegel (1999) was identified by Schore as being instrumental in integrating current cognitive neuroscience into this new developmental model. Schore quotes Siegel as saying, "we need to expand the area of 'affective neuroscience' if we are to meaningfully explore the neurobiological basis of interpersonal relationships, subject experience, and the developing mind" (2001, p. 2). Siegel's work has been instrumental in creating an understanding of the biological constructs of the developing infant brain that manifests in behavioral outcomes like attachment and temperament. Siegel points out that "the brain has been implicitly seen as a 'knower' of the world, as a socially isolated organ whose purpose is to grasp the inanimate world outside it” (Schore, 2001, p. 2). Shore points out that the validity of Siegel's statements is being verified by current research findings in developmental neuroscience that suggest that the development of the infant's emotional brain is directly and actively influenced by his transactions with the animate social and not the inanimate physical environment (Panksepp, 1998; Schore, 2001; Siegel, 1999).

The second researcher Schore cites as he describes the development of his psychoneurobiological theory is John Bowlby (1969). Bowlby is the founder of a long-held interdisciplinary perspective which outlines the processes of early development and the implications this early brain development has on later mental health. His work is rich in findings related to attachment and infant/care giver behavior. Although the empirical work of Bowbly was done in the 1960s, the findings and implications of his work are relevant today. Schore used the theories derived from Bowlby's (1969) research to create a holistic view of infant mental health development. Of particular interest to Shore was Bowlby's hypothesis that suggests that the developmental processes of an infant are the product of the interaction of a unique genetic endowment with a particular environment of adaptiveness, especially of his/her interaction with the principal figure in that environment, namely his/her mother and that the infant's capacity to cope with stress is correlated with certain maternal behaviors. Bowlby (1969) also suggested that there is a correlation between attachment theory that could frame a heuristic hypotheses about the etiology and neurophysiology of psychiatric disorders (Schore, 2001). Schore points out that it is clear that, as Bowlby was attempting to shed light on the theory surrounding infant attachment, he was also suggesting a neurological foundation - a foundation that was undetectable with the technology of his time.

With the advancement of technology and further research, Schore (2001) has been successful in identifying significant components of a new developmental theory that incorporates behavior, biology, and environment. In recent years, Bowlby (1969) responded to Schore's work by suggesting that the current developmental conceptions that integrate the psychological and biological realms are bringing us closer to a complex biopsychosocial model that can serve as a source of not only the next level of questions for science but also pragmatic applications of this knowledge. He added that these deeper investigations of infant mental health, of the primary forces that impact the development of human nature, can do more than alter the intergenerational transmission of psychpathology. They can significantly increase the numbers of individuals who posses an intuitive sense of emotional security, and thereby the quality of life of the infant, child, and adult members of our society.

In his article, Schore (2001) pulls together the work of Siegel (1999) and Bowlby (1969) to offer an overview of his interdisciplinary perspective of infant brain development. In his description, Schore outlines a connection between infant brain development, attachment theory, stress regulation, and infant mental health. He points out that research findings indicate that the relationship between infant brain development and social responses are found to develop architecturally in the right hemisphere of the developing brain.

Researchers have been helpful in identifying the structures and functions of the right brain. They have found that the right brain develops the most during the first three years of life, and that it is centrally involved in processing social-emotional information, facilitating attachment function, and regulating bodily and affective states (Schore, 
2001). Schore further points out that the right brain is also critical in the control of vital functions supporting survival and enabling the organism to cope actively and passively with stress. Schore (2001) has found, through his research, that these regulatory systems are experience dependent and that the experience is embedded in attachment relationships between the infant and the primary caregiver. Schore's psychoneurobiological model suggests that there is a link between secure attachment, development of efficient right brain regulatory function, and adaptive infant mental health.

Schore is not alone in his assumptions about the developing brain. Michael Johnson (Johnson, 2000), a researcher for the Neurological and Pediatric Department at Johns Hopkins University, suggests that the human infant brain undergoes remarkable organizational changes during intrauterine and postnatal life. According to Johnson, the success of these organizational changes relies on the intricate dance of the neurological system. The neurological system is made up of a variety of cell types. The most significant of the cells being the nerve cells called neurons that were described in detail earlier by Pally (1997). According to neurobiologists like Johnson (2000) and Diamond (1988), each of those 100 billion neurons has the capability to generate a chemical response that is significant to the overall function of the brain. As mentioned before, there are terminals at the base of each of the axons. These terminals house a myriad of chemicals called neurotransmitters. Neurotransmitters work with the neurological system to send messages that work to regulate the neural system. Some familiar neurotransmitters are: dopamine, serotonin, adrenaline, and cortisol.

Neurotransmitters are released from a terminal and enter an available receptor on a branch of a dendrite. This transmission is based on information input through the senses. There is a reticular activator at the base of the brain stem that acts as a switching station between information input and chemical release. As the body experiences sensory information, the brain identifies the activation needed on a chemical level. A specific chemical (neurotransmitter) will be released and will in turn activate a physiological response in the body. As an example, if a person smells smoke while sitting in his/her living room, his/her brain assesses the appropriateness of the smoke in the living room. The information about the smoke enters the brain system via the olfactory senses. The brain then determines the chemical reaction that best facilitate the survival of the person. The neurotransmitter, adrenaline will be released from the terminals of the axons and enter receptor sites on the branches of dendrites. There will then be a physiological response. The presence of adrenalin will initiate an increased heart rate, more blood will be sent to the muscles, and the person will have a heightened sense of alertness. Due to the chemical response, the person will, physiologically, have the ability to get help and possibly put out a fire.

According to Johnston (1995), neurotransmitters are present in the developing brain while still in utero. His research indicates that the developing brain is very responsive to these chemicals and the presences of them in the developing brain have been tied to maternal behavior.

The next section of this paper will discuss one of the neurotransmitters that is commonly implicated as critical for the appropriate function of the developing brain. The neurotransmitter is cortisol, known as glucocorticoids. It has many functions in both the developing and mature brain.

\section{THE ROLE OF CORTISOL IN BRAIN DEVELOPMENT AND FUNCTION}

In describing the significance of cortisol, I refer to the work of Eliner-Avishai, Brunson, Sandman, and Baram (2002) which provides insight into neurochemicals and the adaptation of organisms. The findings from their work with primates is widely accepted and translated to human development. According to Eliner-Avishai et al. (2002), the ability of an organism to adapt to its environment is integral to its survival. Eliner-Avishai et al. point out that daily life involves confrontations with changing situations that can be physiologically or psychologically challenging. To cope with these actual or perceived threats of stress, the ability to alter function and expression of neuronal genes has been developed in the form of molecular and behavioral stress responses. Eliner-Avishai et al. also suggest that this is advantageous because it allows rapid behavioral, autonomic, and cognitive responses to stressful circumstances, followed by the prompt re-establishment of the functional steady state. This means that upon sensing stress, our brain not only initiates rapid secretion of effected molecules (noradrenalin and adrenal glucocorticoids) but also responds to the inciting signal with patterned and coordinated changes in programmed gene expression. 
Adrenal glucocorticoids (cortisol in humans), are stress hormones also known as catecholamine and are known to form the first line of defense for mammals (including humans) under conditions that threaten homeostasis (i.e., conditions of stress) (Gunnar \& Barr, 1998). Gunnar and Barr describe glucocordicoids as the end products of the hypothalamic-pituitary-adrenal (HPA) system (Gunnar \& Barr, 1998). It has been determined that glucocorticoids help to mobilize and distribute energy stores, influence the activity of the immune system and coordinate adaptive behaviors in the brain (Gunnar \& Barr, 1998; deWeerth \& van Geert, 2002). Given these findings, Gunnar and Barr describe (1998) cortisol, and the process that regulates it is part of the story of how early experiences shape brain development.

Many researchers have contributed to our understanding of the function of cortisol by citing findings from both human and animal studies. One of the most prolific of those researchers is M.J. Meaney. (1996) added clarity to our understanding of the role of cortisol with respect to early brain development by discussing findings from both animal and human research. The findings from the animal research suggest that early experiences "program" the brain's stress circuits in ways that affect later cognitive competence, emotional responding, and activity of physiologic systems that orchestrate reactions to stress and challenge. Meaney continues his discussion about early brain development and chemical reactivity by citing research that was performed with preterm infants. The research findings identified prenatal treatments with synthetic glucocordicoids offered great benefit to preterm infants. However, animal studies of prenatal synthetic glucocorticoids exposure indicate that there may be some long-term physiological costs of early exposure to excess glucocordicoids. Further, the effects may not become apparent until later in life (Meaney, 1996). Caspi et al. (2002) found that some male children who are maltreated grow up to develop antisocial behaviors while others do not. Their findings suggest that a functional polymorphism in the gene encoding the neurotransmitter-metabolizing enzyme monoamine oxidase was found to modulate the effects of maltreatment. In addition to these research findings, studies in rodents and primates suggest that responsively and regulation of the hypothalamic-pituitary-adrenal (HPA) system later in life may be shaped by social experience during early development. Although it has been determined that cortisol is a major hormonal product of the HPA system in humans, more information about its influence is needed. Current research has provided a partial understanding of the activity of this neuroendocrine axis. It is speculated that the regulation of this system may bear importantly on human growth and development. This development may be influenced by biological as well and environmental factors (Matthews, 2002; Gunnar, 1998; de Weerth, 2002).

The environment must be taken into account when considering the postnatal development of the infant's brain. The ability of the early environment to program the HPA axis has been documented in several species. There is considerable evidence that a similar process can also occur in humans. Studies of animals indicate that the phenotype of HPA function follows early manipulation, which depends on the timing and intensity of the manipulation, in addition to the gender of the fetus or neonate (Matthews, 2002). Matthews has found that there is considerable interplay between the HPA and the hypothalmo-pituitary-gonadal axes, and emerging evidence indicates that this interaction is modified by early environmental manipulation. Studies are rapidly unraveling the mechanisms that underlie developmental programming of the HPA axis (Matthews, 2002). Matthews (2002) states that understanding these mechanisms could hold the key to the development of therapeutic interventions aimed at reversing the impact of an adverse intrauterine of neonatal environment.

According to deWeerth et al. (2002) research in recent years has seen an upsurge in studies of cortisol response. deWeerth et al. suggests that this is occurring for two reasons. The first reason is that cortisol has been found to be a hormone with many different and important functions such as: the regulation of the energy system, the activation of the immune system, and support of the cognitive system. In addition, cortisol has been found to play an important role in stress response. It is known to be secreted when an organism faces a difficult or problematic situation that is perceived as stressful (deWeerth, et al, 2002).

The second reason for the interest in this area is related to advancements in laboratory instrumentation and techniques that have occurred in the last decade. deWeerth points out that previously cortisol levels could only be measured in serum or urine. Currently cortisol can be easily measured and reliably assessed in saliva using fairly cheap, ready made kits (Schwartz, Douglas, Susman, Gunnar, and Laird, 1998). There is consensus that this simple measure provides a window on the psychobiology of the stress response through which those who study children may 
observe the interacting effects of biological, contextual, historical, temperamental, and behavioral factors (Schwartz et al., 1998).

An example of work that has evolved from this advancement comes in the form of a phenomenon described by Gunnar and Vazquez (2001) termed hypocortisolism. The two researchers start their description of hypocortisolism by saying that for many years we have known that stress has been associated with increased activity of the limbichypothalamic-pituitary-adrenocortical (LHPA) axis. They go on to say that recent studies in adults have shown that this neuroendocrine axis may be hyporesponsive in a number of stress related states (Gunner, 2001; Vazquez, 2001). This phenomenon is described as a paradoxical suppression of the LHPA axis under conditions of trauma and prolonged stress. They apply this hypothesis to early development by saying that they believe that the experience of adverse conditions early in life are thought to contribute to the development of hypocortisolism in adulthood. In describing the ramifications of this phenomenon, the researchers point out that low cortisol levels may not be an indication of low stress but may need to be investigated further to identify the possibility of hypocortisolism due to early experiences of undue stress.

deWeerth (2002) conducted research in this area using cortisol measurement instruments in an attempt to shed some light on some profound facts about infants and their mothers. By measuring cortisol levels in infants using salivary samples, deWeerth was able to find that, before birth, human infants have an established basal cortisol level. According to deWeerth (2002), early in life cortisol is secreted in a pulsatile fashion by the adrenal cortex and its levels show a strong circadian rhythm, being lowest around midnight and highest in the early morning hours. She has found that after birth, the infant's basal cortisol level decreases linearly with age and is negatively related to sleep (deWeerth, 2002). deWeerth et al. looked further into cortisol levels in infants and found that while infants showed relative stability across individuals they displayed great intra-individual variability across assessments. The mother's cortisol levels were also assessed. They found that unlike the infants, the mothers display inter-individual variability, together with a relative stability across assessments. It was also found that the infant's intra-individual variability was not affected by gender, or time of visit, nor was it related to the mother's basal cortisol. Daily measures of basal cortisol taken in a subgroup of infants indicated the day-to-day intra-individual variability to be of the same magnitude as the week to week variability.

The works of Gunnar (2001) and deWeerth (2002) have been made possible due to the advancement of technology and the increased availability of a variety of research measures. Determining the appropriate measure has become an issue when considering research design. Schwartz (1998) suggests that the appropriateness of the cortisol measure may be at least in part based on the research question.

In 1999, a group of 14 researchers contributed to the further understanding of these measures by gathering to share their expertise in the areas of chemical reactivity and brain function. Their discussions attempted to identify appropriate instruments, strategies, and protocol for measuring salivary cortisol. The researchers were unable to identify one measurement instrument as superior but were in agreement regarding the need to determine the instrument based on the research direction. Despite the nebulous nature of instrument selection, there was a clear protocol established for testing identified by the research group. That protocol included the description of the appropriate process of sampling by suggesting that a minimum of four or five samples of saliva should be taken in a day. It was also stated that the measures should be taken one, four, nine, eleven hours after waking. The group of researchers suggests that the morning and evening cortisol samples are the most important and that the other three or four samples may be random. One group of researchers, Schwartz et al. (1998) suggests that the data should be collected over three to four days to get a reliable assessment of a "trait" daily concentration and for six or more days. The 14 researchers that make up the MacCarthur Research Network (1999) have settled on a one-day, six-sample protocol. The timing of the samples is: 1) awakening 2) 45 minutes after awakening 3) 2.5 hours after awakening 4) 8 hours after awakening 5) 12 hours after awakening, and 6) bedtime. When working with an infant population, the collection times would need to be adjusted to accommodate a shorter awake time. The MacCarthur Network also identified some control factors to consider when using these measures. They are: the stable characteristics of the individuals like age and gender, state characteristics which include menstrual cycles, contraceptives, and other medications, chronic disease, dynamic characteristics like food intake, sleep status, exercise, wake time, smoking, and 
finally physiological characteristics (Seeman, 2000). Participant protocol is outlined in three steps by the MacArthur Network as follows:

- $\quad$ Participants fill out a log during the day that the samples are being collected using the MacArthur Salivary Cortisol protocol.

- $\quad$ Participants fill out a log book about how many controls they experienced (smoking, exercise, drinking, stress of the day) 3. Participants are interviewed about the stress of the day in comparison to other days they have recorded (Stewart, 1999).

Cortisol is only one piece of the biological puzzle. It is, however, a piece that can provide insight into the inter-functioning of the developing human brain. The current measurability of neurochemicals like cortisol along with the growing understanding of the profound implications of these chemicals will lead researchers to further their investigations in this area. The findings from future research will have a profound impact on our understanding and subsequent treatment of infants. It will answer questions for researchers like Gunnar and Barr (1998) who profoundly identified the research phenomenon by stating that cortisol and the process that regulates it are part of the story of how early experiences shape brain development.

\section{BREAST FEEDING: BIOLOGICAL AND SOCIAL FACTORS}

For many infants one of those early experiences is breast feeding. Cortisol has been found in breast milk and is presumed to be passed to the infant during feedings. Because cortisol is also known to influence brain activity, we can assume that there is a correlation between breast feeding and brain development with cortisol acting as a structural agent. As mentioned in part two, when looking at infant brain development, we need to consider the influences of the environment. Breast feeding is without question a significant environmental factor. Significant findings in the area of breast feeding and brain development have been reported in recent studies by Mezzacappa, Tu, and Myers (2002), Rolf and Keil (2002), Nelson and Panksepp (1998), and Fleming, O’Day, and Kraemer (1999). The studies have examined the sociological, nutritional, and biological impacts of breast feeding on human and animal offspring.

An example of animal research that has provided significant data that was transferred to human constructs was conducted by Cook (1997). Cook specifically investigated the neuropeptide cortisol using sheep as a subject group. In his work with sheep, Cook found that the cortisol response to stress appears to differ between lactating and non-lactating animals. He found that lactating animals had a slightly higher basal level of cortisol and a lower cortisol response to stress than their non-lactating counterparts.

Another research team that uses both animals and humans to better understand human brain development is led by Elizabeth Mezzacappa (Messacappa, Tu, and Myers, 2002). Using the findings of researchers like Cook, Mezzacappa's research team has shed light on the issue of breast feeding as an environmental construct in human infants. In their research, which was conducted using human infants, the research team generated data that suggests that breast feeding reduces maternal subjective and physiological responses to stress and negative moods (Mezzacappa et al., 2002). The findings also indicate that weaning appears to increase maternal subjective and physiological responses to stress. To compliment these findings Mezzacappa et al. also cites research that has identified differences in mood and perceived stress using breast feeding subjects. These studies indicate that breastfeeding buffers the physiological responses in the laboratory. In analyzing these findings, data from the breast fed subjects were compared to the bottle fed subjects. The findings from this comparison indicated that breast fed infants had lower baseline electrodermal conductance and less heart rate reactivity to stimuli. The same study also compared breast and bottle feeding in relation to cortisol levels and found that breast fed infants had lower cortisol levels when introduced to an exercise experience. This finding suggests that the breast fed infants had a blunted hypothalamicpituitary-adrenal (HPA) axis response to stress.

Fleming et al. (1999) provided more meaning to this phenomenon by reporting findings from research that was conducted in an attempt to identify the sensory, endocrine, and neural mechanisms that underlie early motherinfant relationship by encompassing findings related to the cues and characteristics that are present during responsive 
mother-infant interactions. One of the areas of study focused on the chemical reactivity present during lactation. The findings from this particular interest area were established using the chemical assay measurement instruments that Schwartz et al. (1998) referenced in their work. One significant finding that emerged from their assay results suggested that both the mothers and their offspring showed enhanced neuropeptides (cortisol, oxytocin and endogenous opioids) during the lactation period (Fleming et al., 1999). These neuropeptides are thought to be instrumental in the responsiveness of the mother and infant to each other. It is also thought that the presence of these neuropeptides are directly related to the presence of affiliate behaviors in the mother-infant pair such as enhanced olfactory function, regulated suckling, and heightened maternal behaviors (Fleming, 1999). Knowing that maternal behavior and infant responsiveness are the precursor to later relationship structures, these findings provide us with a biological view of bonding and attachment. The findings also make clear the notion that behaviors that facilitate the structure of the mother-infant relationship are directly correlated to the chemical production in the maternal and infantile biological systems (Fleming et al., 1999). Fleming et al. (1999) suggest that these findings indicate that early experiences and associated brain changes clearly affect how infants interact with the world in general, and with their own offspring as adults. Fleming et al. (1999) say that establishing exactly how the neurobiological changes produced by early experiences might persistently alter the neurobiological mechanisms providing for later maternal learning and plasticity constitutes the next challenge.

Nelson and Panksepp (1998) have also been instrumental in identifying the components of research that are significant to our understanding of the biological impacts of breast-feeding. Nelson and Panksepp's research used rats to study the biological substrates of attachment and provided another segue from animal research to human implications. The researchers investigated maternal behavior, sexual behavior, social memory, and the role of neurochemicals. In determining the significance of each of these research areas Nelson and Panksepp investigated the role feeding had on infant development and subsequent attachment. They suggested that milk plays at least some role in the formation of attachment in mammalian infants. They go on to say that milk transfer is one of the primary functions of the mammalian infant-mother relationship and that milk infusion has been shown to induce a dramatic behavioral activation in rat pups (1998). They go on to mention that milk transfer is an important part of regulating sleep-wake, and arousal cycles, and has been shown to induce odor preferences in rat pups. They suggest further that milk transfer is likely to contribute to the funneling of behavior toward the mother and as a stimulus around which an infant's behavior is organized (1998).

When considering the significance of the biological substrates of mammalian milk, Nelson and Panksepp (1998) indicate that there is considerable evidence that suggests that the neurochemical systems are physiological components of these sensory domains in infant rats and are a core part in the physiological regulation of mother directed behaviors. Based on these findings Nelson and Panksepp believe that attachment and affiliative behaviors displayed by rats and other mammalian infants may represent the emergence of adult affiliative brain systems and the ontogeny of a core attachment/affiliative motivational system in the brain.

Koletzko et al. (2001) took the work of Nelson and Panksepp and applied their findings from rodent studies to human milk. The research of Koletzko et al. (2001) focused primarily on the nutritional substrates of human milk, identifying that human milk from healthy well nourished mothers is the preferred form of feeding for all healthy newborn infants and adds that the nutrients found in human milk support normal growth and development in infants. Because the focus of their research was primarily nutritional substrates of human breast milk, they describe in detail the composition of human milk related to vitamins, fatty acids and other nutritional components. Koletzko et al. (2001, p. 3), describe the existence of these nutritional substrates as precursors to what they call the "indispensable structural components of cellular membranes" and suggest that they are deposited to a great extent in the growing brain and retina during perinatal development.

Through the research endeavors of people like Fleming (1999), Mezzacappa (2002), Nelson and Panksepp (1998), and Kolezko et al. (2001), we now have a better understanding of the role breast feeding plays in social, cognitive, and biological development. These findings have contributed to the field of early childhood studies by generating empirical data that has led to broader thinking in the area as well as the generation of more questions to be answered in future research. One area of research that has generated more questions for further research is the existence of neurochemicals like cortisol in mammalian milk. Using what is currently known about the significance of 
these neurochemicals in mammalian milk researchers can further investigate the relationships between cortisol, breast feeding, and infant behavior.

\section{THE STUDY OF INFANT BEHAVIOR}

Infant behavior is a broad field of study that has a rich research history. This history has led to many categorical views of infant behavior. The most common categories are infant temperament, attachment, and regulation. Each of these categories has significant and unique views of infant development. Each of these categories also offers significant insight into the developmental process by which infants assimilate to the social world they enter at birth.

Nathan Fox and Heather Henderson (1999) have studied infant behavior and suggested that infant behavior is synonymous with temperament. They define infant temperament as behavioral styles that appear early in life and are a direct result of neurobiological factors (Fox \& Henderson, 1999). They state (1999) that temperament is thought to describe those aspects of behavioral responding that are not due to interactions with caregivers or other environmental influences, but rather those aspects that are present from birth and are biologically based. They believe that individual differences in young infants' reactivity are a result of the excitability of the central nervous system that can be measured using autonomic, endocrine, and central nervous system changes in response to stimulation.

Jerome Kagan (1996) has a similar model in which he suggests that temperamental inhibitions in infants vary in the degrees of their reactivity to novelty and mild stress. He feels that this reactivity is a function of the excitability of certain nuclei within the amygdala and surrounding regions such as the central gray region of the brain.

Davis, Hitchcock, and Rosen's (1987) work with rats brought clarity to our understanding of the neurological function of the developing brain that describe the neural circuits and structures of the brain stem and mid-brain and are associated with the animals' heightened fear responses as a result of conditioning, and are universally understood to be translated to human behavior. Davis et al. (1987) and LeDoux (1989) report that the neural systems involved in the heightened fear responses are measured during a classical conditioning paradigm with rats involving a tone or light and shock. According to their findings, this system appears to underlie both the behavioral (e.g., startle, freezing, and negative vocalization) and physiological (e.g., increased heart rate, and stress hormones) signs of fear demonstrated in rats (Fox \& Henderson, 1999). LeDoux argues that human fear reactions originate from the same neural system (Kagan, 1996; Fox \& Henderson, 1999).

The study of infant temperament has long been viewed in tandem with infant attachment behavior. Mary Ainsworth began work in this area in the 1950s and has generated volumes of research findings that indicate that there is a correction between infant temperament and infant attachment behaviors. Colleagues such as Bowlby (1969) and Lewis et al.(2000) who have continued Ainsworth's work have agreed that there is a relationship between infant temperament and social attachment. Lewis et al. (2000) suggest that the concept of attachment has captured our attention because it seeks to explain, in part, the origins of social and emotional behaviors. Lewis et al. say (2000, p. 707) that attachment theory has generated an enormous amount of theoretical and empirical work. The work suggests attachment is essentially an infant's early overt behavior that later becomes a representations of the interpersonal experience he/she had. It is thought that a child's model of attachment relationship is organized around the history of the caregiver's response to the infant's actions. It is also thought that the infant's behaviors are the overt representations of the cognitive process that he/she do not yet have language to support.

This historical work has led to the identification of attachment categories. These categories were determined by using a behavior measurement instrument called the Ainsworth Strange Situation Test. The Ainsworth Strange Situation Test is thought to capture overt behaviors that reflect an infant's internal working model of attachment relationships. Hertsgaard, Erickson, and Nachmias (1995) describe the process by which the Ainsworth Strange Situation is administered. They describe the measure as a cumulative stress paradigm test consisting of seven, threeminute episodes, including two separations and two reunions. The results of the measurements are determined by observing sets of behaviors that move the individual toward and maintain proximity with an attachment figure (Lewis 
et al. 2000). Four principle attachment patterns have emerged from this empirical research. It is thought that these patterns reflect and predict important aspects of social development (Cassidy \& Berlin, 1994).

The attachment patterns are: secure, avoidant, resistant/ambivalent, and disorganized/disoriented attachment. Secure attachment is characterized by the infant's willingness to explore rooms and toys with interest in preseparation episodes. The infant shows signs of missing the parent during separation, often crying by the second separation. The infant shows an obvious preference for the parent over the stranger and greets the parent actively, usually initiating physical contact. There is usually some contact maintaining by second reunion, but then settles and returns to play (Siegel, 1999). Avoidant attachment is characterized by the infant's failure to cry on separation from the parent. The infant actively avoids and ignores the parent on reunion (i.e., by moving away, turning away, or leaning out of arms when picked up). There is little or no proximity or contact seeking, no distress, and no anger. The response to the parent appears unemotional. The infant focuses on toys or the environment throughout procedure. Resistant or ambivalent attachment is characterized by an appearance of distress even prior to the separation, with little exploration. There is a preoccupation with the parent throughout procedure. The infant may seem angry or passive. The infant fails to settle and takes comfort in parent on reunion, and usually continues to focus on the parent and cry. The infant also often fails to return to exploration after reunion (Siegel, 1999, p. 74). The last attachment behavior is called disorganized. This attachment pattern is characterized by the infant displaying disorganized or disoriented behaviors in the parent's presence, suggesting a temporary collapse of behavioral strategies. For example, the infant may freeze with a trance-like expression. The infant may rise at the parent's entrance, then fall prone and huddle on the floor; or may cling while crying hard and leaning away gaze averted (Siegel, 1999, p. 74).

In recent discussions about this long-held view of attachment, researchers have questioned the foundational principles on which this theory of attachment was established. Mangelsdorf, McHale, Diener, Goldstein, and Lehn (2000) suggest that we need to look at Ainsworth's attachment theory through new lenses. They indicate that the Strange Situation procedures have more to do with endogenous characteristics of the infants than to the sensitivity of maternal care. Mangelsdorff et al. (2000) suggest that there are three explanations of the association between attachment and temperament. The first explanation is that temperament and attachment are orthogonal constructs (temperamental variations may influence aspects of behavior but not the overall organization of security at attachment). The second explanation is that temperament may determine what type of insecurity (avoidant or resistant) the child manifests, and the third explanation is that relationship history totally transforms temperamental variations that consequently make little or no contribution to the relationship. Although the Ainsworth model of attachment is still held in high regard, there are other models emerging based on the Strange Situation model but with new views, like those of Mangelsdorff et al.(2000) and others who bring modern measure for future analysis.

Rothbart and Hwang (2002) describe temperament as the individual differences in emotional and motor reactivity and in the attentional capacities that support regulation. Rothbart and Hwang are leading experts in the area of infant behavior assessment and have suggested that the ability to measure infant behavior has increased during the past century. Measures have been created that have allowed early temperament to be empirically linked to later personality, the development of conscience and empathy, as well as the development of behavior problems (Rothbart \& Hwang, 2002). Rothbart has been a pioneer in the development of these behavior measures with the development of the Infant Behavior Questionnaire (Gartstien \& Rothbart , 2003I) which is designed to measure infant temperament using parent report. Following the original development and implementation of the IBQ, Rothbart redesigned the instrument to included nine new scales and minor modifications. The revised IBQ (IBQ-R) (Gartstien \& Rothbart , 2003) measures three broad areas of personality development. They are surgency/extraversion, negative affectivity, and orienting/regulation. The Rothbart measurement instrument is widely used and highly regarded as a valid instrument for the measurement of infant behavior.

The theory of infant temperament that expands on the work of Fox and Henderson (1999) and Kagan (1996) is described in an article by Schore (2001) entitled, "Effects of Secure Attachment Relationships on Right Brain Development, Affect Regulation, and Infant Mental Health.” In his article, Schore identifies infant temperament by using a psychoneurobioloical theory of infant brain development. Recognizing the work of neurobiologists, Schore introduces the concept of infant regulation. Schore describes infant regulation as integration of both the biological and the psychological realms. Schore states (2001) that because the concept of regulation is a theoretical concept 
that is shared by many clinical and basic sciences it can be used to further develop models of normal and abnormal structure. He states that this interdisciplinary research and the clinical data affirm the concept that in infancy and beyond, the regulation of affect is a central organizing principle of human development. Although the concept of regulation is not a new one, it takes on new elements when viewed through Schore's model. The model introduces the role biology plays in infant regulation. To support this, Schore cites Damasio (1994) as saying "Emotions are the highest order direct expression of bioregulation in complex organisms” (Schore, 2001, p.10). The connections between infant brain development and the significance of infant regulation of the development of infant mental health structures is pointed out by Damasio (1994), who notes that the overall function of the brain is to be well-informed about what is going on in the rest of the body proper, about what goes on in itself, and about what the environment surrounding the organism, so that suitable survivable accommodations can be achieved between the organism and the environment. The psychobiological model incorporates components from the maturational theory and components from the cognitive developmental theory by looking at how the biological architecture of the developing brain work together with environmental conditions to establish patterns of infant regulation.

Schore's (2001) psychoneurobiological theory is supported further by the research of Gunnar and Donzella (2002) that specifically identified the role that cortisol plays in early brain development. This theory of infant regulation is supported by citing animal studies suggesting that responsivity and regulation of the biological system later in life may be shaped by social experience during early development. They indicate that cortisol is found to play a part in the regulation of the biological system in humans. Although it provides only a partial understanding of the activity of this neuroendocrine axis, its regulation may bear importantly on human growth and development. Gunnar and Donzella (2002) identify developmental studies of cortisol and behavior in children, birth through age five and describe the development of the social buffering of cortisol responses. They also established a functional analogue of the rodent stress hyporeponsive period by the time children are about 12 months of age. They further describe the sensitivity of cortisol activity to variations in care quality among infants and toddlers, along with evidence that children with negative emotional temperaments may be most likely to exhibit elevations in cortisol under conditions of less-than-optimal care.

Regulation is identified by Gunnar and Donzella (2002) in reference to the activity in the limbic hypothalamic-pituitary-adrenocortical system. Gunnar and Donzella (2002) cite the past research findings of S. Levine from 1994 and Suomi from 1991. The work of Levine and Suomi was conducted using rodents and primates. Like other studies, the research findings are universally translated to be used as human behavior predictors. Gunner states (2002) that Levine (1994) and Suomi (1991) have identified that caregivers play an important role in regulating activity in the biological system during development. In rodents, licking and grooming by the dam and the delivery of milk into the gut maintain the adrenal hyporesponsive period, a period between postnatal day four through the fourteenth day. During this time, it is difficult, yet not impossible, to elevate glucocordicoids levels (i.e., cortisol). Gunnar and Donzella (2002) discuss the findings of Graham and Heim (1999) who translated animal findings to human behavioral predictors in their paper "Effects of Neonatal Stress on Brain Development: Implications for Psychopathology.” In their paper, they cited a theoretical review that suggested that adverse experiences early in life predispose individuals to affective pathology in part through affecting activity of the biological system. Although this research has identified a strong correlation between treatment early in life and the biological development of the brain in animals, there is little information about these issues in human infants.

The study of infant behavior is a complicated area of research. Defining infant behavior is difficult; equally difficult is identifying the appropriate measurement of the phenomenon. Whether the researcher is viewing infant temperament as behavioral, or regulatory there are common characteristics found in the investigations. Biology, social interactions, and environmental influences are woven throughout all of these works. There are many opportunities for further research in this area. Future findings will shed light on the complexities of the biological, social, and psychological development of infant behavior.

\section{SUMMARY}

In order to identify the impact breast feeding has on the developing brain as well as infant behavior, this review investigated four separate yet interconnected disciplines: brain development, how cortisol interacts during 
brain development, the role breast feeding plays in brain development, and the subsequent development of infant behavior. Brain development is thought to be a delicate biological interplay of genetics and environment. Research suggests that the brain undergoes a surge of development during the first five years of a child's life. Optimal development was been found to require a balance of nutritional, social and environmental supports.

The second area investigated was the stress hormone cortisol. Cortisol was found to be present in developing brain and was also found to play an important role in infant brain development and behavioral regulation. Cortisol was also determined to be present in maternal breast milk. The relationship between the presence of cortisol in breast milk and the impact it has on the feeding infant is yet to be studied but has interesting implications for the identification of maternal stress response and infant reactivity.

The third area of study was the impact breast feeding has on infant brain development. Human milk has been found to have a positive impact on the development of the brain as well as qualities that strengthen the developing immune system. The emotional benefits were also identified. It is clear from a number of research sources that the social interaction required for breast feeding positively contributes to infant-mother bonding process.

The final area of study was infant behavior. Behavior was found to be an offshoot of all of the developmental constructs that take place during infancy. Researchers agree that infant behavior, although somewhat nebulous in its nature, is biologically, socially, and environmentally driven.

What has been identified through this investigation of literature is that there are clear relationships between brain development, chemical reactivity in the brain, breast feeding, and infant behavior. The scope of these relationships is still unclear but within the realm of further research.

\section{REFERENCES}

1. Ainsworth, M.D. \& Bowlby, J. (1991). An ethological approach to personality development. American Psychology, 46, 333-341.

2. $\quad$ Bowlby, J. (1969). Attachment and loss Vol. 1 attachment. New York: Basic.

3. $\quad$ Brazelton, T.B. (1973). Neonatal behavior assessment scale. Clinical Development Med, 50.

4. $\quad$ Carnegie Foundation. (1995). Starting Points, Carnigie Foundation, N.Y.

5. Cassidy, J. \& Berlin, L. J. (1994). The insecure/ambivalent pattern of attachment: theory and research. Child Development, 65 (4), 971-991.

6. Damasio, A.R. (1994). Descarte's Error. New York: G.P. Putnam’s Sons.

7. Davis, M., Hitchcock, J.M. \& Rosen, J.B. (1987). Anxiety and the amygdala: pharmacological and anatomical analysis of the fear potentiated startle paradigm. The Psychology of Learning and Motivation, 21, 263-305.

8. deWeerth, C. \& van Geert. (2002). A longitudinal study of basal cortisol in infants: intra-individual variability, circadian rhythm and developmental trends. Infant Behavior and Development, 152, 1-24.

9. $\quad$ Diamond, M. \& Hopson, J. (1988). Magic trees of the mind. New York: Penguin.

10. Dubowitz, L. M., Cowan, F., Rutherford, M., Mercuri, E., Pennock, J. (1995). Neonatal neurology, past present and future: A window on the brain. Brain and Development, 17, 22-30.

11. Eliner-Avishai, S., Brunson, K. L., Sandman, C. A. \& Baram, T. Z. (2002). Stressed-out in utero? Trends in Neurosciences, 25 (10), 518-524.

12. Essex, M.J., Klein, M. H., Cho, E., \& Kalin, N.H. (2002). Maternal stress beginning in infancy may sensitize children to later stress exposure: Effects on cortisol and behavior. Biological Psychiatry 52, 776-784.

13. Fleming, A. S., O'Day, D.H., \& Kraemer, G.W. (1999). Neurobiology of mother-infant interactions: Experience and central nervous system plasticity across developmental and generations. Neuroscience and Behavioral Reviews, 23, 673-685.

14. Fleming, A. S., Steiner, M., \& Cortner, C. (1997). Cortisol, hedonics, and maternal responsiveness in human mothers. Hormones and Behavior, 32, 85-98.

15. Fox, N., \& Henderson, H.A. (1999). Does Infancy Matter? Predicting social behavior from infant temperament. Infant Behavior and Development, 22 (4), 445-455. 
16. Gartsein, M. A., \& Rothbart, M.K. (2003). Studying infant temperament via the revised infant behavior questionnaire. Infant Behavior and Development, 166, 1-23.

17. Graham, F.K. (1956). Behavioral differences between normal and traumatized newborns. Psychol Monographs: General and Applied, 70.

18. Granham, Y.P. \& Heim, C. (1999). The effects of neonatal stress on brain development: Implications for psychopahthology. Developmental Psychopathology, 11, 545-565.

19. Gunnar, M. R., \& Donzella, B. (2002). Social regulation of the cortisol levels in early human development. Psychoneuroendocrinology, 27, 199-220.

20. Gunnar, M. R., \& Barr, R.G. (1998). Stress, early brain development, and behavior. Infants and Young Children, 11(1), 1-14.

21. Gunnar, M.R., Vazquez, D.M. (2001). Low cortisol and a flattening of expected day time rhythm: potential indices of risk in human development. Development and Psychology, 13, 515-538.

22. Gunner, M.R., Brodersen, L., Nachmias, M., Buss, K., \& Rigatuso, J., (1996). Stress reactivity and attachment security. Developmental Psychobiology, 29 (3), 191-204.

23. Hertsgaard, L. G., M.R., Erickson, M.F., \& Nachmias, M. (1995). Adrenocortocal responses to the strange situation in infants with disorganized/disoriented attachment relationships. Child Development, 6 (4), 11001106.

24. Johnson, M. (2000). Functional brain development in infants: Elements of an interactive specialization framework. Child Development, 71(1), 75-81.

25. Johnston, M. V. (1995). Neurotransmitters and vulnerability of the developing brain. Brain and Development, 1995 (17), 301-306.

26. Kagan, J. (1996). Three pleasing ideas. American Psychologist, 51, 901-908.

27. Kagan, J. (1994). Galen’s prophecy: Temperament in human nature. New York: Basic Books.

28. Koletzko, B., Rodriguez-Palmero, M., Demmelmair, H., Fidler, N., Jensen, R., \& Sauerwald, T. (2001). Physiological aspects of human milk lipids. Early Human Development, 66(Supplement), 3-18.

29. LeDoux J. (1989). Cognitive-emotional interactions in the brain. Cognition and Emotion, 3, $267-289$.

30. LeDoux, J. (2002). The synaptic self, Viking Press, N.Y.

31. Lewis M., \& Feiring, C., \& Rosenthal, S. (2000). Attachment over time. Child Development, 71(3), 707-720.

32. Levine, S. (1994). The ontogeny of the hypothalamic-pituitary-adrenal-axis: The influence of maternal factors. New York Academy of Science, 4, 275-288.

33. Lupien, S.J., King, S., Meaney, M.J., \& McEwen, B.S. (2002). Child's stress hormone levels correlated mother's socioeconomic status and depressive state. BiologicalPsychiatry. 48, 976-980.

34. Mangelsdorf, S. C., McHale, J.L., Diener, M.,Goldstein, L.H., \& Lehn, L. (2000). Infant attachment: Contributions of infant temperament and maternal characteristics. Infant Behavior and Development, 23, $175-196$.

35. Matthews, S.G. (2001). Antenatal glucocorticoids and the developing brain: Mechanisms of action. Seminars in Neuroatology, 64 (4), 309-317.

36. Matthews, S.G. (2002). Early programming of the hypothalamo-pituitary-adrenal axis. Trends in Endocrinology \& Metabolism.

37. McArthur, S., Shah, A.N., Morris, J.F., Flower, R.J., Buckingham, J.C., \& Gillies, G.E. (2003). Programming effects of neonatal dexamethasone treatment on the ventral tegmental area in adulthood: The potential role of annexin 1. program no. 149.4. 2003 Abstract Viewer/Itinerary Planner. Washington, DC: Society for Neuroscience.

38. Meaney, M. J. (1996). Early environmental regulation of forebrain glucocrodicoid receptor gene expression: Implications for adrenocortical responses to stress. Developmental Neuroscience, 18, 49-72.

39. Mezzacappa, E.S., Tu, A.Y, \& Myers, M.M. (2002). Lactation and weaning effects on physiological and behavioral response to stress. Psychology and Behavior, In press.

40. Neil G. (1997). Nutrition and cognitive function. Brain and Development 19, 165-170.

41. Nelson, E. E., \& Panksepp, J. (1998). Brain substrates of infants-mother attachment: Contributions of opioids, and norepinephrine. Neuroscience and Behavioral Reviews, 2(3), 437-452.

42. Pally, R. (1997). How brain development is shaped by genetics and environmental factors. International Journal of Psychoanalysis, 78, 587-593. 
43. Panksepp, J. (1998). Affective neuroscience: The foundation of human and animal emotion. New York: Oxford University Press.

44. Paterson, R., \& Moran, G. (1988). Attachment theory personality development and psychotherapy. Clinical Psychology Review, 8, 611-638.

45. Posner, M. I. (2001). Developing brains: The work of the Sackler Institute. Clinical Neuroscience Research, 258-266.

46. Putnam, S.P., Ellis, L.K., \& Rothbart, M.K. (2001). The structure of temperament from infancy through adolescence. In A. Eliasz \& A. Angleitner (Eds.) Advances in Research on Temperament 165-182. Germany: Pabst Science.

47. Prechtl, H.F. (1977). The neurological examination of the full-term newborn infant: A manual for clinical use. Clinical Development Med, 63.

48. Rolf, B., \& Keil, W. (2002). Investigation of oral swabs taken from newborn infants after breastfeeding. Legal Medicine, 4, 52-54.

49. $\quad$ Rothbart M.K. (2001). Emotion and temperament. Developmental Science, 4, 313-329.

50. Rothbart, M. K., \& Hwang, J. (2002). Measuring infant temperament. Infant Behavior and Development, 25, 113-116.

51. Schore, A. N. (2001). Contributions from the decade of the brain to infant mental health: An overview. Infant Mental Health Journal, 22(1-2).

52. Schore, A.N. (2001). Effects of a secure attachment relationship on brain development, affect regulation and infant mental health. Infant Mental Health Journal, 22, 345-375.

53. Schwartz, E. B. G., Douglas A., Susman, E. J, Gunnar, M. R., \& Laird, B. (1998). Assessing salivary cortisol in studies of child development. Child Development, 69(6), 1503-1513.

54. Seeman,T. (2000). Salivary cortisol. Retrieved from http://www.macses.ucsf.edu/Research/Allostatic/notebook/salivarycort.html.

55. Siegel, D. (1999). The developing mind: Toward a neurobiology of interpersonal experience. New York: The Guilford Press.

56. Thomas, A. \& Dargassies, S.A. (1952). Etudes neurologique du tonus masculaire. Paris:Flammarion.

57. Worobey, J. (1989). Temperament rating at 2 weeks, 2 months, and 1 year differential stability and activity and emotionality. Developmental Psychology 8, 257-263. 


\section{NOTES}

